\title{
Gestión de carreras contemporáneas: desafíos críticos para la gestión humana
}

\author{
Contemporary careers management: \\ critical challenges for human resource \\ management
}

Gestão de carreiras contemporâneas:
desafios críticos para a gestão de pessoas

Carlos Fernando Torres Oviedo*

Fecha de recepción: 18 de julio de 2014

Concepto de evaluación: 22 de septiembre de 2014

Fecha de aprobación: 28 de noviembre de 2014

\footnotetext{
Profesor ocasional del Departamento de Administración y Organizaciones de la Facultad de Ciencias de la Administración, Universidad del Valle, Cali, Colombia. Doutorando em Administração, Programa de Pós-Graduação em Administração, Escola de Administração -PPGA/EA- Universidade Federal do Rio Grande do Sul -UFRGSBrasil. Magíster en Psicología con énfasis en Psicología Organizacional y del Trabajo, Universidad del Valle, Cali, Colombia. Administrador de Empresas, Universidad del Valle, Cali, Colombia. Correo electrónico: carlos.f.torres@ correounivalle.edu.co
} 


\section{Resumen}

Con el presente artículo de revisión se exponen algunos lineamientos conceptuales sobre carreras en organizaciones. Se hace especial énfasis en la transformación que esta práctica ha sufrido, dadas las nuevas condiciones del trabajo generadas por los cambios tecnológicos, económicos y culturales que se han venido presenciando desde finales del siglo XX. La literatura especializada muestra, en el presente momento histórico, un escenario más dinámico y complejo para las personas y las organizaciones. Se encontró que en las condiciones actuales, las personas dirigen su propia carrera con el propósito de alcanzar mejores niveles en sus trayectorias dentro o fuera de la organización. Ello plantea nuevos retos y maneras de pensar la gestión de personas, a fin de generar prácticas alternativas. En último término, a modo de sugerencia, se plantean algunas cuestiones para ser abordadas por los responsables en gestión humana, en el sentido de construir relaciones recíprocas con las que se contribuya al desarrollo mutuo.

Palabras clave: carrera, gestión de carrera, gestión humana.

Clasificación JEL: M53, M11, M12

\section{Abstract}

This review article lays out some conceptual guidelines regarding careers in organizations. Special emphasis has been made on the transformation that this practice has experienced since the late twentieth century, given the new working conditions created by the technological, economic, and cultural changes that have taken place. The literature shows a more dynamic and complex stage for people and organizations in the current historical moment. It was found that, under the current conditions, people guide their own careers, aiming at achieving higher levels within and/or outside the organization. This poses new challenges and ways of thinking about managing people, leading to the development of alternative practices. Finally, as a suggestion, some questions to be addressed by those responsible of human management are presented, aimed at building reciprocal relationships that contribute to mutual development.

Keywords: career, management career, human resource management. 


\section{Resumo}

Este artigo de revisão expõe alguns lineamentos conceituais sobre carreiras em organizações. É feita uma ênfase especial sobre a transformação que esta prática tem sofrido, dadas as novas condições de trabalho geradas pelas mudanças tecnológicas, econômicas e culturais que têm se apresentado desde o final do século XX. A literatura especializada amostra, neste momento histórico, um panorama mais dinâmico e complexo para as pessoas e organizações. Verificou-se que, nas condições atuais, as pessoas dirigem sua própria carreira, procurando alcançar melhores níveis em suas trajetórias no interior e/ou fora da organização. Isso cria novos desafios e formas de pensar acerca da gestão de pessoas, a fim de gerar práticas alternativas. Enfim, como uma sugestão, surgem algumas questões a serem abordadas pelos responsáveis da gestão de pessoas, no sentido de construir relações de reciprocidade que possam contribuir ao desenvolvimento mútuo.

Palavras-chave: carreira, gestão de carreira, gestão de pessoas. 
No tiene sentido contratar personas inteligentes y después decirles lo que tienen que hacer. Nosotros contratamos personas inteligentes para que nos digan qué tenemos que hacer.

Steve Jobs

\section{INTRODUCCIÓN}

El siglo XXI ha traído una serie de nuevas circunstancias para las organizaciones, derivadas de la incorporación de las tecnologías de la "teleinformación" en reemplazo de la tecnología "electromecánica" (Malvezzi, 1998, p. 1). La competitividad global ha presionado para replantear las acciones de las organizaciones de acuerdo con el nuevo escenario, en los planos productivo, financiero y de mercadeo. El área de gestión humana tampoco escapa a este reto. Gestionar personas en el contexto histórico actual, exige ponerse a tono con las implicaciones derivadas de los cambios globales.

Reconociendo la importancia de la gestión humana como factor estratégico en las organizaciones (Calderón, 2004; García, Murillo \& González, 2011), vale la pena preguntarse por las posibilidades de acción que se pueden llevar a cabo en el interior de las mismas. En este orden de ideas, resulta necesario problematizar algunas categorías clave -tales como carrera- para ampliar nuestra comprensión acerca del mundo de las organizaciones y del trabajo (Rentería, 2009).

Con el presente documento -a modo de revisión teórica- se plantea examinar y discutir el concepto de carrera en las actuales condiciones del mundo del trabajo y las organizaciones, a fin de formularse algunos interrogantes que orientarán posibles acciones para emprender desde el área de gestión humana ${ }^{1}$. Para ello se llevó a cabo un proceso de revisión

\footnotetext{
Es importante aclarar que el presente artículo toma aportes teórico-conceptuales sobre las carreras en organizaciones. Para estudios empíricos sobre el tema, el lector podrá consultar los estudios de Núñez-Cacho, Grande-Torraleja \& Pedrosa-Ortega (2012); Lips-Wiersma \& Hall (2007); Ivancheva \& Gourova (2011); Arthur, Khapova \& Wilderom (2005); Whymark \& Ellis (1999), entre otros.
} 
documental sobre textos teóricos acerca de la gestión de carreras. Se aplicó análisis de contenido, en la perspectiva de Bardin (2002), considerando categorías como: 1) cambios históricos en la concepción de carrera, 2) tipologías de carrera, y 3) carreras y gestión de personas.

De este modo se logró recopilar y organizar un material que da cuenta de varios de los ángulos desde los que se puede estudiar el fenómeno de las carreras en el mundo contemporáneo. Se acudió al modelo de carrera académica (Baruch, 2011) a fin de vislumbrar algunas alternativas para discutir. En último término, se presenta un repertorio de posibles acciones por emprender en las organizaciones, con el fin de dirigir las carreras de las personas, en función del desarrollo tanto de la organización como de ellas mismas, orientado por un ideal de reciprocidad.

\section{CONTEXTUALIZACIÓN}

Conceptos como organización, trabajo, carrera, gestión de personas, contrato psicológico, compromiso, identidad, entre otros, están siendo discutidos desde finales del siglo pasado, dadas las nuevas condiciones generadas a partir de los cambios técnico-económicos, políticos, sociales y culturales que han impactado a las organizaciones (Rentería \& Torres, 2012; Kilimnik, 2011; Dutra, 2010; Veloso \& Dutra, 2010; Ribeiro, 2009b; Rentería, 2008; Dent \& Whitehead, 2002;
Malvezzi, 2001; Arthur \& Rousseau, 1996b). Varios de estos autores resaltan la necesidad de replantear los repertorios que se han ido construyendo alrededor del fenómeno organizacional, por cuanto están fundamentados en aquellas grandes organizaciones del sector industrial, que existían en entornos relativamente estables. Por eso es necesario acudir a las nuevas discusiones que intentan ampliar los horizontes de la comprensión, para centrarse en la actual diversidad de tipos de organizaciones $y$, por lo tanto, sobre lo referido a las carreras.

Ribeiro (2009a) sostiene que el concepto de carrera está ligado a diferentes disciplinas y enfoques teóricos utilizados para aproximarse al fenómeno desde principios del siglo XX. En ese sentido, propone un análisis histórico de las concepciones de carrera en dos períodos: 1) desde 1900 hasta la década del 70 y, 2) desde "1970 hasta los días de hoy" (Ribeiro, 2009a , p. 143). Durante el primer período, los estudios de carrera estuvieron centrados en la gran organización tipo industrial, caracterizada por asignar tareas rutinarias en el marco de una estructura jerárquica, dentro de la cual las personas escalaban según los criterios de asensos determinados por la dirección de gestión humana ${ }^{2}$. En ese momento, las ciencias de la gestión (administración y psicología organizacional tradicional), entendían la carrera como el recorrido de los individuos dentro de la estructura organizacional en un sentido continuo y ascendente.

2 En adelante: D-GH 
Durante el segundo período, en el llamado contexto "posmoderno", caracterizado por múltiples formas organizativas, mediadas por las tecnologías de la información, así como por la orientación al mercado cambiante y dinámico, los estudios de carreras están dedicados a comprender las diversas posibilidades que personas y organizaciones están experimentando entre sí, alrededor del fenómeno del trabajo. En ese sentido, hoy se concibe la carrera como el recorrido de las personas en múltiples organizaciones a lo largo de su vida laboral, considerando tanto las opciones como las condiciones que encuentran en el mercado de trabajo, ahora más complejo y cambiante. De manera que, para las ciencias de la gestión, la carrera pasa a ser entendida como un movimiento determinado por el individuo sin que necesariamente pase por etapas predefinidas, vinculado no solo a una organización, a través de sus competencias y capacidad de aprendizaje. Por otra parte, para las ciencias del trabajo (psicología social del trabajo y las organizaciones y la sociología del trabajo) la carrera es una construcción formada en la relación individuo-procesos organizativos, que genera estructuras colectivas y experiencias individuales. Así, la carrera no sería más una estructura predeterminada sino "un proyecto social en construcción conjunta con el proyecto de vida de cada persona, siempre en una dinámica relacional" (Ribero, 2009a , p. 146).

De este modo, se plantea la presente reflexión en el marco del nuevo escenario, que demanda otras aproximaciones que orienten las acciones organizacionales. Para ello es necesario retomar lo enunciado por algunos autores sobre el concepto de carrera.

\section{APROXIMACIONES AL CONCEP- TO DE CARRERAY PERSONA QUE TRABAJA}

En términos disciplinares, la carrera ha sido abordada desde diferentes ópticas. Ribeiro (2009a) plantea que la psicología organizacional tradicional ha entendido la carrera como un vehículo de autorrealización personal. Por su parte, la sociología la ha visto como el desempeño de papeles y la movilidad en los sistemas sociales. Por otra parte, la psicología social la asume como el fenómeno de ajuste, interacción o relación dialéctica entre individuo y sociedad. Otras contribuciones se encuentran en la antropología, que estudia el estatus social; la economía, que observa las respuestas ante las fuerzas del mercado; la ciencia política, que está centrada en las estrategias de poder; y la historia, que aporta a la comprensión de las construcciones autobiográficas.

Respecto a los enfoques epistemológicos, de acuerdo con Ribeiro (2009a), se tienen cinco visiones. Desde el enfoque esencialista, el individuo nace con unos rasgos que le facilitan su acceso al trabajo (Holland, 1973). El desarrollo de la carrera se facilita en la medida en que la persona "se conoce" para "explotar" su potencial, el cual ya posee. El punto de vista interaccionista plantea que el individuo es a partir de su relación con 
la sociedad (Schein, 1978). La carrera consistiría en los comportamientos de la persona en el trabajo a partir de las influencias que recibe de su medio más próximo. Desde la mirada funcionalista, el individuo se adapta a las condiciones sociales (Savickas, 1997). La carrera estaría determinada por las acciones que se emprenden para ajustarse a los requerimientos del trabajo. En la visión dialéctica, el individuo y la sociedad se afectan mutuamente (Arthur, Hall \& Lawrence, 1989). La carrera estaría basada en las negociaciones que se llevan a cabo entre personas y organización en una dinámica de poderes. Finalmente, para la perspectiva construccionista, los individuos interpretan discursivamente su lugar en el espacio social cotidiano (Young \& Collin, 2004). Aquí la carrera hace parte de las elaboraciones individuales -mediadas por lo colectivo-, acerca de lo que es y podría significar el trabajo como factor determinante de la propia realidad.

Ribeiro (2009a) propone cinco maneras posibles para el análisis de carreras. Los análisis estructurales se enfocan en la escala jerárquica del organigrama organizacional (Hall, 1976); los tipológicos se dedican a establecer clasificaciones y modelos (Super, 1957; Chanlat, 1995; Kanter, 1997; Dutra, 2001); los desarrollistas estipulan etapas o fases que describen el proceso de maduración de las carreras (Schein 1993); los transicionales consideran los cambios y las rupturas que experimentan las personas cuando van de una organización a otra o cambian de ocupación (Hopson \& Adams, 1977); y los análisis psicosociales estudian la interacción entre personas y organizaciones (Super, 1957; Baruch, 2004; Bourdieu, 1974), resaltando el carácter dinámico y la posibilidad que tiene cada una de las partes para influir en la otra.

A efectos del presente documento, se parte de la perspectiva dialéctica centrada en análisis psicosocial, desde donde se concibe la carrera como el itinerario o recorrido que las personas llevan a cabo en múltiples escenarios organizacionales a lo largo de su trayectoria laboral. En este orden de ideas, autores como Arthur y Rousseau (1996a) plantean que actualmente la carrera está en manos de las personas, dado que en el mercado laboral (así como en el interior de las organizaciones), transitan de un lugar a otro, construyendo su propia trayectoria de modo individual. En ese sentido, ya no es el encargado de la D-GH quien diseña la carrera de los empleados de la organización. En cambio, son las mismas personas las que ahora toman decisiones acerca de lo que consideran más apropiado para sus proyectos de vida. De este modo asistimos a lo que Beck y BeckGernsheim (2002) han llamado el proceso histórico de individualización, entendido como el fenómeno contemporáneo en el que cada quien quiere ser autor de su propia biografía ${ }^{3}$.

\footnotetext{
Sin embargo, Rentería (2012) advierte que las condiciones personales y socio-históricas de empleabilidad, no son las mismas para todas las personas. Tener esto presente resulta importante, dado que en los discursos de responsabilización individual, sobre el ingreso al mercado laboral, se legitiman prácticas de exclusión social.
} 
Desde el mismo punto de vista psicosocial, algunos autores (Kilimnik, 2011; Baruch, 2011; Dutra, 2010; Veloso \& Dutra, 2010; Ribeiro, 2009b; Rentería, 2008; Dent \& Whitehead, 2002; Malvezzi, 2001; Arthur \& Rousseau, 1996a) han venido planteando que en la organización contemporánea, las personas están sacando provecho de su relación con las organizaciones, en el sentido de capitalizar sus aprendizajes y relaciones, que alimentan lo que Schein (1993) denomina "carrera interna". Así, nos encontramos ante el nuevo trabajador contemporáneo, al que Malvezzi (1998) llama el "agente económico reflexivo", y al cual describe como el profesional que ha incorporado la crítica y la hermenéutica como sus principales insumos de trabajo. Todo esto genera interrogantes nunca antes planteados para la D-GH, en el sentido de que ahora las personas también deciden donde y en qué condiciones trabajan, entendiendo que su paso por la organización quizás sea de manera temporal, es decir, tan solo un momento dentro de su carrera individual.

Asumir esta mirada permitiría reflexionar sobre -por lo menos- dos implicaciones para la D-GH. En primer lugar, su foco de atención sobre las personas, como por ejemplo sus formas de interaccionar, modos de relacionamiento, identidad y significados que construyen en torno al trabajo, como lo plantean Rosso, Dekas y Wrzesniewski (2010) y Wrzesniewski, Dutton y Debebe (2003). En segundo lugar, sobre la valoración de las fortalezas individuales en función del trabajo. Al respecto, aportes desde la "psicología positiva" (Linley, Harrington \& Garcea; 2010), podrían ofrecer elementos interesantes acerca de la posibilidad de identificar y optimizar los recursos personales positivos en contextos laborales.

En este orden de ideas, asistimos a lo que Bendasoli (2009) denomina como una recomposición en las relaciones de las personas con su propio trabajo. Cabe ahora el interrogante por la comprensión de esta nueva realidad, por parte de los encargados de la gestión humana en las organizaciones. Y más aún, ¿qué podría hacer la D-GH para gestionar -o contribuir con- la carrera de las personas que trabajan?

\section{ALGUNAS CONTRIBUCIONES}

La revisión de la literatura retomada hasta el momento, ha sugerido que las organizaciones están inmersas en una nueva dinámica que plantea, por una parte, las presiones de la competitividad global (tensiones externas), y por otra, las fuerzas de las personas que trabajan (tensiones internas). De este modo, habría que reconocer que las personas han llegado a este nuevo escenario con sus proyectos de vida individuales, comprometidas no solo con una organización sino con su propia trayectoria, en múltiples direcciones y con la capacidad de renovar sus recursos de trabajo (conocimiento, competencias, redes de relaciones), para ponerlos al servicio de quien consideren como el más acorde con sus expectativas. Según lo mencionado hasta ahora, hay que preguntarse ¿Cuáles son las acciones que se 
pueden sugerir a la D-GH para gestionar procesos de carrera en las actuales condiciones? ¿Cómo podrían conciliarse los intereses individuales con los organizacionales? ¿A cuál modelo de "hombre" nos enfrentamos?

Baruch (2011) plantea que una de las alternativas para entender y gestionar las carreras en las organizaciones contemporáneas, está relacionada con el modelo de carrera académica ${ }^{4}$. En ese sentido, Baruch y Hall (2003) afirman que este modelo puede operar como un "prototipo de guía" (Baruch, 2011, p. 20), un indicador de dirección y de cambio en los sistemas de carrera en otros sectores. En esta perspectiva, podrían encontrarse insumos interesantes para la gestión de carreras, basados en la movilidad interna, el apoyo a la formación permanente, la valoración por méritos de producción y no de tiempo, la rotación de puestos, la actualización constante, entre otros, como aspectos que configuran las llamadas carreras multidireccionales (Baruch, 2003).

En este nuevo panorama de carreras, la D-GH podría asumir algunos desafíos para afrontar la nueva dinámica de las carreras, partiendo de una perspectiva dialéctica en la que individuos y organización se influyen entre sí, acuerdan condiciones y, de este modo, se transforman mutuamente. En la historia de la gestión humana, no estamos acostumbrados a negociar sino tan solo a asignar condiciones y responsabilidades. Acudimos a la razón instrumental para obtener ideas de las personas para incrementar la productividad o ampliar el mercado, pero poco para negociar las condiciones que faciliten el desarrollo de la carrera. Por lo tanto, a continuación se proponen algunas cuestiones encaminadas a la reflexión -y la acción- de los interesados en temáticas relacionadas con la dirección y gestión de personas. No conforman una fórmula universal, en cambio, representan solo un conjunto de sugerencias, ya que en cada organización se presentan condiciones diferentes:

- Redimensionar la concepción de carrera: resignificarla en un sentido más amplio, admitiendo que la promesa de trabajo para toda la vida es muy difícil de sostener (para ambas partes) y que, por lo tanto, las personas están en permanente búsqueda de mejores condiciones dentro y fuera de las organizaciones.

- Reconocer el nuevo perfil de los trabajadores: admitir que las personas han construido en torno a sus aspiraciones individuales, competencias y conocimientos que pueden aportarle a la organización más allá de los estándares mínimos de desempeño esperado en cada cargo.

\footnotetext{
Sobre el concepto de carrera académica, Baruch (2011) plantea que una posibilidad para gestionar procesos de gestión humana en las organizaciones empresariales, en las actuales condiciones de dinamismo, sería la del modelo universitario. En ese sentido, podría retomarse la idea de movilidad interna, que incluye nociones como temporalidad en cargos, promociones por méritos en la productividad, apoyo a la formación, entre otras, como política de gestión de carreras.
} 
- Aprovechar las nuevas capacidades: disponerse para el beneficio de las posibilidades creativas, propositivas e innovadoras que poseen todos los seres humanos en las organizaciones contemporáneas, a través de la participación en proyectos, comités u otras formas organizativas temporales.

- Generar condiciones para el trabajo autónomo: proveer los medios (técnicos y administrativos), para centrar las labores en resultados, en lugar de hacerlo en el control autoritario, típico de la vieja organización tipo industrial.

- Brindar herramientas técnicas para la eficiencia: dedicar los instrumentos necesarios para mejorar los procesos administrativos, a fin de que el trabajo se agilice y dé lugar al tiempo para la gestión y socialización del conocimiento.

- Generar sistemas de compensación alternativos: proponer y negociar estrategias de incentivos y beneficios más flexibles y personalizados, que estimulen las capacidades de las personas dentro y fuera de la organización.

- Favorecer espacios de diálogo: emplear mecanismos para el establecimiento de acuerdos de trabajo, acordes con las necesidades individuales de las personas, tales como: mejoramiento de la relación trabajo-familia y procesos de formación, capacitación y entrenamiento.

- Facilitar recursos: suministrar insumos financieros, formativos y técnicos para afrontar el mundo del trabajo, en caso de que las personas deban gestionar su propia empleabilidad, ${ }^{5}$ dentro o fuera de la organización, por despido, jubilación o decisiones de emprendimiento, a partir de los cuales se pueden establecer alianzas estratégicas y de asesoramiento.

- Establecer criterios de carrera flexible y multidireccional: formular pautas basadas en logros individuales, relacionados con los aportes a la organización, en lugar de decidir la promoción interna con fundamento solamente en el tiempo de permanencia en el cargo.

- Permitir la incorporación de personas jóvenes: considerar, hasta donde sea posible, las capacidades potenciales de los trabajadores que apenas inician su propia carrera profesional, reconociendo que el ser humano tiene la posibilidad de aprendizaje y que esto contribuiría al alcance de objetivos de ambas partes.

- Promover valores de solidaridad y apoyo mutuo, en vez de la competitividad individualista, para construir ambientes más cooperativos.

Todo esto implicaría transformar los significados de quienes lideran procesos de gestión humana, en lugar de delegar trabajo a las personas, bajo la ideología de la flexibilización laboral, multifuncionalidad, colaboración y lealtad. En este orden de ideas, la D-GH

\footnotetext{
5 Al respecto, Mateos (2014) plantea que parte del compromiso social de las organizaciones y el sistema productivo en general, es justamente fomentar la empleabilidad y el bienestar de sus trabajadores. En ese sentido, se asume que como consecuencia de la reestructuración y carácter dinámico del mercado laboral, las organizaciones deben facilitar procesos de desarrollo a través de un modelo pedagógico de formación en la empresa.
} 
tiene el reto de transformar su mirada sobre las nuevas condiciones (externas e internas) en las que hoy se encuentran las organizaciones. De este modo, sus acciones podrían estar más acordes con las de las personas que las conforman.

\section{CONCLUSIONES}

El concepto clásico de carrera anclado a una sola organización, a través del cumplimiento paulatino de criterios establecidos por la D-GH, se ha ido superando con miras a una noción de carrera multidireccional, o carrera sin fronteras, en la que las personas mismas son las que ahora dirigen sus trayectorias, en función de sus propios intereses dentro o fuera de la organización.

Actualmente, desde una perspectiva dialéctica y una aproximación psicosocial, es posible reconocer que la persona puede incidir en las decisiones organizacionales en materia de gestión humana, para quizás ser la autora de su propia biografía. Por ello, el encargado de la dirección de gestión humana debería considerar lo que se ha denominado como "carrera subjetiva", estimando el proyecto de vida laboral que las personas construyen para sí mismas. Esto implica replantearse la concepción tradicional de "hombre", para apreciar de manera más amplia las posibilidades que pueden ofrecer las personas dentro de la organización, aunque sea de manera transitoria. Sin embargo, el reto no termina allí. Además de la revisión de lo que se entiende por persona, trabajo, carrera y organización, es necesario que las acciones emprendidas contribuyan también al desarrollo de las trayectorias individuales $\mathrm{y}$, de esta manera, perfilar relaciones de reciprocidad.

Las cuestiones planteadas, lejos de pretender formularlas como prescripciones aplicables de modo general a cualquier tipo de organización, buscan generar discusión en torno a lo que podría hacerse en el nuevo panorama de las carreras. Dado que, tradicionalmente, la gestión humana ha pretendido cambiar a las personas en función de los objetivos de la organización, valdría la pena cuestionarse las prácticas que se llevan a cabo para replantear formas de relacionamiento. Tal vez es hora de invertir la ecuación, reconociendo que actualmente las personas cuentan con capacidad de discernimiento acerca del lugar en el que podrían ofrecer su potencial.

El quehacer del responsable de la dirección de gestión humana respecto a las carreras, podría ser un interesante objeto de investigación, para comprender cómo se está afrontando actualmente la pretensión de las personas de alcanzar mejores niveles en sus trayectorias. Futuras investigaciones podrían enfocarse en los repertorios que se han incorporado, y que podrían incluir su visión sobre el trabajo, la nueva organización, la gestión de personas y, por supuesto, la carrera, con los cuales orientan sus acciones en nuevo escenario del siglo XXI. 


\section{REFERENCIAS}

1. Arthur, M. B., Hall, D. T. \& Lawrence, B. S. (eds). (1989). Handbook of career theory. Cambridge: Cambridge University Press.

2. Arthur, M.B., Khapova, S.N. \& Wilderom, C.P.M. (2005). Career success in a boundaryless career world. Journal of Organizational Behavior, 26 (2), 177-202.

3. Arthur, M. B. \& Rousseau, D. M. (1996a). The boundaryless career as a new employment principle. In M. G. Arthur \& D. M. Rousseau (Eds.). The boundaryless career (p. 3-20). New York: Oxford University Press.

4. Arthur, M. \& Rousseau, D. (1996b). A Career Lexicon for the 21st Century. Academy of Management Executive, 10 (4).

5. Bardin, L. (2002). Análisis de contenido ( $3^{\mathrm{a}}$ ed.). Madrid: Akal.

6. Baruch, Y. (2003). Career systems in transition: a normative model for career practices. Personnel Review, 32 (2), 231-251.

7. Baruch, Y. (2004). Transforming careers: from linear to multidirectional career paths organizational and individual perspectives. Career Development International, 9 (1), 58-73.

8. Baruch, Y.(2011). Transformacões nas Carreiras: De trajetórias lineares para multidirecionais Perspectivas organizacionais e individuais. En Z. Kilimnik (ed.). Transformacões e transicões nas carreiras, estudos nacionais e internacionais sobre o tema. Rio de Janeiro: Qualitymark.

9. Baruch, Y. \& Hall, D.T. (2003). Careers in academia as role model for career systems, Journal of Vocational Behavior, 64(2), 241-262.

10. Beck, U. \& Beck-Gernsheim, E. (2002). Individualization. London: Sage.

11. Bendassolli, P. (2009). Recomposição da relação sujeito-trabalho nos modelos emergentes de carreira. Revista de Administração de Empresas, 4 (49), 387-400.

12. Bourdieu, P. (1974). Economia das trocas simbólicas. Sáo PauIo: Perspectiva.

13. Calderón, G. (2004). Lo estratégico y lo humano en la dirección de las personas. Revista Pensamiento y Gestión, (16), 158-176.

14. Chanlat, J. F. (1995). Quais carreiras e para qual sociedade? Revista de Administração de Empresas, 35 (6), 68-75.

15. Dent, M. \& Whitehead, S. (2002). Introduction. In Managing professional iden case study on managing the new career during organizational change. Journal of Organizational Behavior 28 (6), 771-792.

16. Malvezzi, S. (1998). El agente económico reflexivo. Revista Desarrollo y Capacitación, XIX.

17. Malvezzi, S. (2001). The building of professional identity and the boundaryless careers. Conferencia no publicada. 
18. Mateos, T. (2014). The meaning of employability in the new labour relationships between company-employee: a model of training in companies. Procedia Social and Behavioral Sciences, 139, 448-455.

19. Núñez-Cacho, P., Grande-Torraleja, F. \& Pedrosa-Ortega, C. (2012). Nuevos retos en el desarrollo de carrera profesional: el modelo boundaryless career. Universia Business Review, (34), 14-35.

20. Rentería, E. (2008). Nuevas realidades organizacionales y del mundo del trabajo: implicaciones para la construcción de la identidad o del sujeto. Informes Psicológicos, $10,65-80$.

21. Rentería, E. (2009). De recursos humanos a la psicología organizacional y del trabajo: reflexiones a la luz de las realidades actuales del mundo del trabajo. En M. Aguilar \& E. Rentería (Comp.). Psicología del trabajo y de las organizaciones: reflexiones y experiencias de investigación. Bogotá: Universidad Santo Tomás.

22. Rentería, E. (2012). Desarrollo de la empleabilidad como estrategia para las organizaciones: limitaciones, posibilidades e implicaciones para las personas. Revista Aristeo, (2), 69-90.

23. Rentería, E. \& Torres, C. (2012). Identidad profesional: proceso de configuración en el caso de dirigentes de organizaciones no gubernamentales. Cadernos de Psicología social do trabalho, 15 (2), 287-302.

24. Ribeiro, M. A. (2009a). Carreira: transformações de uma concepção na teoria e na pratica. En M.A. Ribeiro. Psicologia e gestão de pessoas: reflexões críticas e temas afins (ética, competência e carreira). São Paulo: Vetor.

25. Ribeiro, M. (2009b). A trajetória da carreira como construção teórico-prática e a proposta dialética da carreira psicossocial. Cadernos de Psicologia Social do Trabalho, 12(2), 203216.

26. Rosso, B.D., Dekas, K.H. \& Wrzesniewski, A. (2010). On the meaning of work: a theoretical integration and review. Research in Organizational Behavior, 30, 91-127.

27. Savickas, M. L. (1997). Career adaptability: an integrative construct for life-span, life-space theory. The Career Development Quarterly, 45 (3), 247-259.

28. Schein, E. H. (1978). Career dynamics: matching individual and organizational needs. Reading, MA: Addison-Wesley.

29. Schein, E. H. (1993). Career anchors: discovering your real values. Amsterdam: Pfeiffer.

30. Super, D. E. (1957). The psychology of careers. New York: Harper y Row.

31. Veloso, E. F. R. \& Dutra, J. S. E (2010). A Evolução do conceito de carreira e sua aplicação para a organização e para as pessoas. Em: Dutra, J. (eds.). Gestão de carreiras na empresa contemporânea. São Paulo: Atlas SA.

32. Whymark, K. \& Ellis, S. (1999). Whose career is it anyway? Options for career management in flatter organization structures. Career Development International, 4 (2), 117-120. 
33. Wrzesniewski, A., Dutton, J.E. \& Debebe, G. (2003). Interpersonal sensemaking and the meaning of work. In Research in Organizational Behavior, vol. 25, (pp. 93-135). New York: Elsevier Science.

34. Young, R. A. \& Collin, A. (2004). Introduction: constructivism and social constructionism in the career field. Journal of Vocational Behavior, 64 (3), 378-388. 